\title{
EXPRESSION KINETICS OF INTERFERON STIMULATED GENES IN ATLANTIC SALMON HEAD KIDNEY CELLS (ASK) NUCLEOFECTED WITH POLY(I:C)
}

\author{
Adriana M S Andresen"1 Tor Gjøen ${ }^{1 \S}$ \\ ${ }^{1}$ Department of Pharmaceutical Biosciences, School of Pharmacy, University of Oslo, Oslo, Norway.
}

\begin{abstract}
Viral diseases are of great concern in fish farming. Close to $20 \%$ of salmon put out to sea are lost during production and a large part of this is due to infections. Oil-adjuvented vaccines are available against some virus, but the efficiency under field conditions have been questioned. Our goal is to test known agonists for human Toll-like receptors (TLRs) in modified nanobeads carriers as adjuvants in different vaccines formulations, against fish viruses. The primary early response to viruses is mediated by interferons (IFNs) and interferon stimulated genes (ISGs). The interferon system is activated through the recognition of pathogen associated molecular patterns (PAMP) by conserved pattern recognition receptors (PRRs), such as TLRs. Poly (I:C) is a synthetic analog of double-stranded RNA (dsRNA) that mimics a viral infection in mammals and it is one of the most thoroughly studied PAMPs with respect to immunostimulatory potential in therapeutics or vaccines.

Before developing a vaccine is necessary to understand more about how the immune system responds to these ligands. We therefore analyzed the expression of some interferon stimulated genes at different time points in Atlantic salmon head kidney cells (ASK) transfected by electroporation with Poly(I:C).

Cells were transfected using Amaxa Nucleofector Kit with $5 \mu \mathrm{g}$ of Poly (I:C) or mock transfected with only buffer. After transfection, cells were plated and incubated for different time points $(0,6,12,24,48$, 72 hours) when samples of RNA were extracted for cDNA synthesis.

Our results shows that there are large differences in the temporal pattern of IFN and ISG expression in salmon cells. QPCR analysis revealed strong upregulation of IFN alpha and TNF alpha during the first 6 hours after stimulation. Both Isg15 and Mx1 showed higher levels, comparing with control, in the first 12 hours and reached a peak at 24 hours after transfection.

Mock transfections using only buffer without adding RNA or DNA also induced upregulation of some of the genes tested, especially TNF alpha. Endoplasmic reticulum (ER) stress induced by transfections was analyzed by a XBP-1 splicing assay analysis, but the results were not consistent. Lipofections and nucleofections are popular techniques for introducing RNA and DNA into cells to study both protein expression and immunity to cytoplasmic nucleic acids. Further studies are therefore necessary to quantify the magnitude and duration of cellular stress caused by these methods to separate their impact on effects mediated via PPRs .
\end{abstract}

\section{KEYWORDS}

Atlantic salmon, Interferon, Nucleofection, Poly (I:C), Toll-like receptor

${ }^{\S}$ Corresponding author. Tel.: +47 22844943; Fax: +47 22854402.

E-mail address: tor.gjoen@farmasi.uio.no 